\title{
Presentación del monográfico La dimensión social de la educación superior: perspectivas internacionales
}

\author{
Claudia Iriarte y Alejandra Montané
}

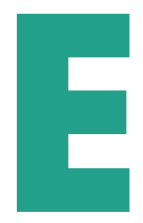

1 presente monográfico de la Revista Sociología de la Educación (RASE) ofrece diversas miradas al tema de la dimensión social de la educación superior, asociadas al proyecto internacional en curso TO-INN From Tradition to Innovation in Teacher Training Institutions financiado por la Unión Europea a través del programa Erasmus, Acción K2 Capacity Building, (573685-EPP2016-1-ES-EPPKA2-CBHE-JP) https://www.toinn.org/ que pretende tender puentes entre la tradición y la innovación en las instituciones de educación superior en América Latina y Europa.

Las inquietudes alrededor del compromiso y la dimensión social en la educación superior que se han compartido en el marco de dicho Proyecto denominado bajo el acrónimo TO-INN, son el origen de este monográfico, el cual tiene como fundamento base la convicción de que la educación es un bien común en el contexto de la sociedad de conocimiento. No en vano, los Objetivos de Desarrollo Sostenible, recogidos en la Agenda 2030 por la ONU, han vuelto a remarcar la necesidad de «garantizar una educación inclusiva, equitativa y de calidad y promover oportunidades de aprendizaje durante toda la vida para todos» (ONU, 2018). En el contexto de la educación superior, este cuarto objetivo está intrincado con la dimensión social de las instituciones universitarias, la cual es una prioridad señalada tanto por la UNESCO en sus Conferencias Mundiales sobre Educación Superior, por los comunicados europeos sobre el Espacio Europeo de Educación Superior y por las Cumbres Iberoamericanas.

TO-INN parte de una visión sistémica de la calidad en Educación Superior en la que la inclusión, el acceso, la supervivencia y el éxito académico de los estudiantes juega un papel relevante aceptando que el proceso de masificación vivido en las universidades a través de los procesos de democratización convive con las desigualdades en muchos aspectos relacionados.

En la propuesta partimos de una conceptualización amplia de la denominada dimensión social inspirada en las conferencias ministeriales de Bergen (2005), Londres (2007), Lovaina (2009), Budapest (2010) y Bucarest (2012), y estudios relevantes sobre la temática que enfatizan la necesidad de conocer el perfil, características y condiciones de los estudiantes como herramienta para el diseño de las políticas que les afectan y con la finalidad de avanzar hacia una educación superior más equitativa e inclusiva.

Esta dimensión es relevante para la promoción de la cohesión social, la reducción de las desigualdades y la elevación del nivel de conocimiento, habilidades y competencias en el centro de la sociedad. Igualmente, se busca incidir en las políticas para poner el acento en maximizar el potencial de las personas en

1 Claudia Iriarte, Universidad Nacional Autónoma de Honduras, claudiar_iriarte@yahoo.com; Alejandra Montané López, Universitat de Barcelona, smontane@ub.edu. 
cuanto a su desarrollo personal y su contribución a una sociedad sostenible, democrática y basada en el conocimiento.

El fundamento e interés que reúne a los autores y autoras en esta aventura editorial no es otro que reconocer, analizar, estudiar, considerar y hacer visibles a los/las estudiantes de la educación superior, sus características y maneras de vivir, sus necesidades e intereses y sus sistemas de participación universitaria.

Una lectura integral de los artículos que conforman el monográfico que se presenta, nos orienta al reconocimiento de que, si bien es cierto, se han logrado grandes avances para el logro de una educación superior equitativa e inclusiva, aun se requieren mayores pasos hacia el conocimiento del perfil, las características y condiciones de vida y participación de los estudiantes como herramienta para el diseño de las políticas que les favorezcan. Asimismo, es preciso profundizar en el análisis del impacto de los procesos de masificación vividos en las universidades y sus resultados, en términos de desigualdades, para potenciar políticas orientadas a la inclusión en el acceso, en la permanencia, en las condiciones de vida y en los resultados académicos de los estudiantes.

\section{Contenido temático del número monográfico}

Este monográfico está compuesto por doce artículos comprometidos con la meta de mejorar la educación superior haciéndola accesible para todos y defendiendo el principio de responsabilidad pública para la educación superior que fue renovado en la Conferencia Ministerial de Bergen (2005), donde también se reiteró la necesidad de establecer condiciones apropiadas para que los estudiantes puedan completar sus estudios sin obstáculos relacionados con su origen social y económico. Sin embargo, este compromiso no puede realizarse si no se visualiza al estudiante como sujeto de ciudadanía y como actor y si no se conoce su perfil, sus características y sus condiciones de vida.

De igual manera, la dimensión y responsabilidad social también abarca las medidas adoptadas por los gobiernos para apoyar a los estudiantes, especialmente a aquellos procedentes de los sectores menos favorecidos, en aspectos financieros y económicos y en proporcionarles servicios de orientación y asesoramiento, a fin de facilitar un acceso más equitativo e inclusivo, basado en el principio de no discriminación. Es por ello, que se requiere una mayor reflexión sobre la cantidad y calidad de dichas medidas, considerando que el conjunto de estudiantes que ingresan, permanecen y culminan la educación superior aún dista mucho de reflejar la diversidad de los pueblos, una de las aspiraciones planteadas en la Conferencia de Londres (2007).

Las reflexiones asociadas a los estudiantes y sus características, así como a la eliminación de las barreras para garantizar el acceso a una educación de calidad e inclusiva, derivan en el primer eje alrededor del cual pivota la primera parte de los artículos contenidos en el monográfico: Características sociodemográficas y composición social.

Por otra parte, existe una conciencia creciente sobre la necesidad de transitar hacia una «educación centrada en los estudiantes y no en una educación centrada en el profeson», (Conferencia Ministerial de Londres, 2007:2), es decir, una educación centrada en el aprendizaje y no en la enseñanza. Desde esta perspectiva, también se dirigen los esfuerzos hacia la eliminación de barreras para el acceso, para la elección de la carrera y para la progresión entre los ciclos y para la implementación de créditos basados en los resultados del aprendizaje y en la carga de trabajo del estudiante. Elementos que se vinculan al segundo eje del monográfico: Elección de estudios y matrícula universitaria. 
De igual manera, es reconocido el rol de las instituciones de educación superior en el desarrollo de las sociedades, así como la demanda de una educación superior basada en la autonomía institucional, la libertad académica, la igualdad de oportunidades y los principios democráticos y se identifican como funciones de la universidad «la preparación de los estudiantes como ciudadanos activos en una sociedad democrática, la preparación de los estudiantes para su futuro profesional y desarrollo personal, la creación y conservación de conocimiento y el fomento de la investigación y la innovación» (Conferencia de Londres, 2007:1).

Estas afirmaciones conducen a analizar el rol de la Universidad ante su responsabilidad social, partiendo en principio de una conceptualización sobre lo que se entiende por dimensión social de la educación superior, así como de un análisis de las relaciones entre la dimensión social y las políticas públicas y de la encrucijada entre investigación, educación e innovación como factores claves del desarrollo humano sostenible, lo que nos encamina hacia el tercer eje alrededor del cual giran las contribuciones: Dimensión social, universidady sociedad.

Asimismo, es reconocida la necesidad de asociar el aprendizaje a lo largo de la vida con la educación superior, con la vida profesional y con el reconocimiento de aprendizajes previos, considerando tanto el aprendizaje formal como el informal o no formal (Conferencia Ministerial de Bergen, 2005). Por otro lado, también se requiere la formación interdisciplinar y el desarrollo de competencias transferibles, según las necesidades del mercado de trabajo, así como la creación de oportunidades para itinerarios flexibles de aprendizaje en la educación superior. Tareas estas, en las que se han logrado importantes avances a través de experiencias e iniciativas tales como la formación por competencias, la certificación de competencias previas, la formación continua a lo largo de toda la vida y la educación a distancia, por mencionar algunos, en los cuales aún queda un largo camino por recorrer. Sin dejar de mencionar que la aplicación de políticas de aprendizaje permanente exige una estrecha colaboración entre las administraciones públicas, las instituciones de educación superior, los alumnos, las empresas y los empleados, tal como se planteaba en la Conferencia Ministerial de Lovaina (2009).

Estas temáticas que se vinculan con los itinerarios para la responsabilidad social universitaria y el desarrollo humano sostenible; el aprendizaje social, los valores y el constructivismo social en el currículo y en la educación superior; el aprendizaje colectivo y la innovación social; la política pública, la ciudadanía digital y la educación como derecho humano orbitan alrededor del cuarto eje abordado en el monográfico: Educación superior inclusiva.

La secuencia de los artículos que se presentan obedece a una clasificación realizada en base a los cuatro ejes anteriormente mencionados: Características sociodemográficas y composición social; elección de estudios y matrícula universitaria; dimensión social, universidad y sociedad; y, educación superior inclusiva. Por lo tanto, el monográfico se divide en cuatro partes.

\section{Características sociodemográficas y composición social}

En este apartado se busca conocer y describir los/las estudiantes de forma tal que las preguntas sobre las características sociodemográficas engloben variables como edad, sexo o pertenencia a pueblos originarios.

En torno al eje características sociodemográficas y composición social situamos tres artículos que abordan la temática de origen, condiciones de vida y participación de las y los estudiantes en la educación superior. 
El artículo Características sociodemográficas y condiciones de vida y estudio de los estudiantes de Educación de origen indígena. La aproximación del proyecto TO-INN, desarrollado por Jordi Méndez Ulrich, Alejandra Montané López, Juan Llanes Ordoñez, Gabriel Hervas Nicolás, Isaac Calduch Pérez, Ricardo Morales-Ulloa, Judith Muñoz Saavedra, aborda desde una definición situacional de los pueblos indígenas en Latinoamérica, la relación teórica y contextual entre los mismos y la educación superior, así como la definición de quienes integran este colectivo. Los elementos presentados en el artículo surgen de un estudio empírico a través de la implementación de un cuestionario a más de doce mil estudiantes de treinta universidades procedentes de diversos países latinoamericanos, cuyos resultados permiten atisbar el perfil sociodemográfico y académico de los estudiantes indígenas pertenecientes a las universidades que integran el Proyecto To Inn, abarcando tanto su contexto familiar, como sus condiciones de vida y participación académica y social. En las conclusiones, los autores destacan que los resultados reflejan una situación menos ventajosa de los estudiantes indígenas y la necesidad de profundizar tanto en el análisis de los datos generados desde el estudio mencionado, como en desarrollar nuevas líneas de investigación que permitan identificar estrategias para asegurar una inclusión real, una participación auténtica y un aprendizaje pertinente para dichos estudiantes.

El artículo Características de los aspirantes a la Escuela Normal en México. ¿En qué condiciones inicia su formación elprofesorado de primaria?, desarrollado por Jihan García-Poyato Falcón, Graciela Cordero Arroyo, desarrolla un estudio cuantitativo con de 345 aspirantes a la Licenciatura en Educación Primaria en universidades de Baja California (México, 2018-2022). El instrumento utilizado fue la encuesta y la información se analizó con distintas pruebas de estadística descriptiva e inferencial. Los resultados presentan las características laborales, socioeconómicas, familiares y académicas del estudiantado, así como se analizan comparativamente las diferencias significativas entre los grupos de aspirantes según se trate de zona urbana o rural. Se concluye en el estudio que los aspirantes de la zona rural se encuentran en desventaja en los tres ámbitos analizados: socioeconómico, familiar y académico.

El artículo Dimensión social del alumnado que cursa formación continua a nivel de posgrado en educación superior en Paraguay: el caso de la Universidad Autónoma de Asunción (UAA) desarrollado por Javier Numan Caballero Merlo analiza la dimensión social en el área de educación de postgrado en la UAA (Paraguay). Se trata de un estudio descriptivo que parte de la aplicación del instrumento de análisis diseñado en el marco del proyecto TO INN. Analiza los resultados en tres dimensiones: a) datos sociodemográficos, b) datos universitarios y d) vida y participación académica y social añadiendo la dimensión motivacional. El texto recoge una información que permite la reconstrucción de los perfiles, estudios, condiciones académicas, vínculos universitarios y motivaciones respecto a su vida y participación académica y social.

\section{Elección de estudios y matrícula universitaria}

Esta segunda parte referida al eje elección de estudios y matrícula universitaria, está constituida por tres artículos donde se abordan los temas vinculados a la equidad participativa y a la composición de la matrícula universitaria, a la elección de los estudios y sus factores asociados, a la igualdad de oportunidades, al riesgo y origen social, a la diversidad y a la relación de autoexclusión e inclusión-exclusión en la educación superior.

El artículo El ingreso a carreras de alta o baja demanda en una universidad mexicana: ¿qué influye en la elección? desarrollado por Mónica López Ramírez, analiza diversos factores asociados a la elección de carrera entre estudiantes de universidades públicas mexicanas, considerando entre ellos las condiciones sociales y 
culturales de origen de los estudiantes y la trayectoria académica previa. El artículo presenta una revisión teórica sobre los efectos de los orígenes sociales en las elecciones y en los resultados educativos y destaca cómo los mecanismos de desigualdad económica, cultural y educativa inciden en la elección de carreras universitarias. Los datos que se exponen provienen de un estudio realizado a los datos generados a través de los instrumentos de ingreso a la UNAM, destacando dentro de los resultados la identificación y caracterización de tres tipos de perfiles académicos de ingreso a la educación superior y la definición e interacción de los factores asociados a la elección de carreras de alta demanda. Para finalizar, el artículo abre las puertas a nuevos estudios y análisis sobre la incidencia de estas variables en la permanencia, rendimiento académico y egreso.

El artículo Diferencias autonómicas en la composición de la desigualdad en la expectativa de matriculación universitaria: efectos primarios y efectos secundarios desarrollado por Manuel Tomás Valdés Fernández parte del interés generado a raíz de los resultados del estudio PISA de 2015 que manifestaba profundas desigualdades en la expectativa de matrícula universitaria del alumnado español de 15 años. En el texto se trata esta desigualdad a partir de la noción de efectos primarios y secundarios del origen social. Cabe destacar que es un interesante estudio que trata las expectativas y enfatiza la importancia en las estructuras de oportunidades del alumnado de distinto estatus socioeconómico y cultural. Todo parece indicar falta de impulso durante las fases educativas obligatorias para impulsar el deseo de cursar estudios superiores en los perfiles de menor estatus económico y cultural. Concluye el autor, que trabajar la dimensión social de la Educación Superior, y especialmente la equidad participativa en la universidad, debe también dirigir los esfuerzos a los periodos educativos previos a la universidad.

En el artículo El carácter contingente de las elecciones y la experiencia universitaria: riesgo y origen social desarrollado por Delia Langa-Rosado, Dani Torrents y Helena Troiano se aborda la experiencia de los estudiantes universitarios de origen social denominado bajo, en contextos de incertidumbre e incremento de las dificultades económicas y de endurecimiento de las condiciones universitarias. El artículo describe el recorrido de las universidades españolas desde la reforma de Bolonia, la crisis económica del 2008 y sus consecuencias en la precariedad laboral y en los cambios derivados en el encarecimiento de las tasas de matrícula, en las titulaciones, en los requisitos de presencialidad, en las condiciones de obtención y retención de becas, en la evaluación continuada y en las modalidades de evaluación. Riesgo, origen social y elecciones educativas se analizan desde la perspectiva de su impacto en las variables de tipo académico, laboral, económico y social. La visión multidimensional del estudio se concentra en las elecciones iniciales, las condiciones de estudio y las perspectivas de futuro de los estudiantes. En el caso de las elecciones iniciales se describe la conexión entre la aversión al riesgo de los estudiantes de origen social bajo y la estructura de derechos percibidos quienes no dan por sentada la posibilidad de continuar estudios universitarios, a diferencia de los jóvenes de origen social medio y alto, quienes sí sienten la apuesta universitaria como un derecho incuestionado. En relación con las condiciones de estudio, se detalla cómo las condiciones de incertidumbre persisten a lo largo de la carrera en los jóvenes de origen social bajo y cómo se trasladan hasta más allá de la obtención del título, lo que podría derivar en procesos de autoexclusión educativa, ya que todas estas variables aumentan el número de obstáculos que los jóvenes deben sortear para acceder, permanecer y culminar la educación superior. 


\section{Dimensión social, universidad y sociedad}

En la tercera parte, se desarrolla el eje de dimensión social, universidad y sociedad a través de tres artículos, que recogen aportaciones teóricas de la dimensión social de la educación superior desde el contexto latinoamericano, las relaciones entre la dimensión social de la educación superior, la democratización en las políticas públicas y el debate entre lo público y lo privado y la provisión de servicios adecuados y útiles a los estudiantes para la eliminación de obstáculos y mejora de la permanencia, retención y deserción estudiantil.

El artículo La dimensión social en la educación superior para las instituciones formadoras de docentes: Una mirada desde Latinoamérica desarrollado por Claudia Iriarte, Ricardo Morales, Lea Cruz, Joseph Malta, Carla Paz, Marlon Medina, Olvis Castro y Rita Tamashiro, presenta una aproximación a la definición teórica de los elementos que conforman la dimensión social en la educación superior para las instituciones formadoras de docentes. Se plantean cuatro subdimensiones de la dimensión social: la igualdad de oportunidades; el origen de los estudiantes; el aprendizaje social y la inserción laboral, movilidad y empleabilidad. En el texto se diserta sobre las políticas inclusivas en la educación superior orientadas al incremento del acceso, a la participación igualitaria para eliminar la discriminación y la desigualdad, al reconocimiento de la diversidad, a las modalidades compensatorias y a la igualdad de oportunidades. Por otro lado, también se destaca la influencia del género, origen étnico, social, cultural y familiar sobre el logro educativo y su impacto en las políticas sociales de discriminación positiva, así como la importancia del aprendizaje individual y del aprendizaje social y colectivo, como parte de la formación integral del estudiante universitario para la construcción de ciudadanía y para la convivencia. La educación superior como factor de movilidad y empleabilidad es abordada, destacando tanto la formación profesional pertinente para ingresar al mundo laboral, como la formación integral sustentada en los principios de la educación para el desarrollo sostenible, el aprendizaje ético y la formación ciudadana. Para finalizar, se destaca la necesidad de contar con políticas públicas educativas coherentes con las demandas profesionales, con la responsabilidad social universitaria y con las demandas de desarrollo de la sociedad.

El artículo Entre lo público y lo privado: acceso y equidad en la educación superior en México y Brasil, 2000-2016, desarrollado por Alejandro Márquez Jiménez y Armando Alcántara Santuario, presenta los avances en cobertura y distribución de las oportunidades de acceso equitativo a la educación superior de dos países: México y Brasil, en el período comprendido del 2000 al 2016. Los resultados presentados destacan que los avances obtenidos en la ampliación de cobertura, no han tenido el mismo ritmo de avance en la reducción de inequidades y asimetrías y en la distribución equitativa de oportunidades de acceso a la educación superior. En el texto se realiza un comparativo entre las estrategias de ampliación de oportunidades de acceso entre los dos países estudiados, resaltando que mientras uno de los países se orienta hacia la ampliación de oportunidades de acceso a través de políticas para expandir la oferta educativa pública, el otro favorece la expansión de instituciones de educación privadas. El artículo transita desde la contextualización y caracterización de los países en análisis y sus políticas afirmativas; pasando por el análisis de tendencias compensatorias a través de becas y por el análisis de la correlación entre variables económicas, sociales, educativas, becas, cobertura y matrícula; hasta la reflexión y el debate sobre lo público y lo privado. Para terminar, los autores concluyen que ha habido un mayor avance en la cobertura de la educación superior en el país donde se ha potenciado la educación superior privada, sin embargo, este incremento en la cobertura no ha impactado en el mejoramiento de la calidad educativa y en los indicadores económicos y sociales. Por otra parte, también señala que en ambos países los programas 
compensatorios continúan siendo insuficientes, por lo que se requiere promover la equidad y el acceso a la educación superior de manera mas contundente y efectiva.

El artículo El rol de la Unidad de Bienestar Estudiantil en la Universidad Estatal del Sur de Manabí, desarrollado por Leopoldo Vinicio Venegas Loor analiza el bienestar estudiantil en el marco de la Educación Superior en Ecuador. La investigación descriptiva se desarrolla en la Universidad Estatal del Sur de Manabí (UNESUM) con el objetivo de describir y analizar los servicios que la universidad ofrece a sus estudiantes. Concluye que las Unidades de Bienestar Estudiantil en las instituciones de Educación Superior, creadas a través de una Ley orgánica, se configuran como un pilar estratégico de la institución.

\section{Educación superior inclusiva}

En la cuarta parte, se aborda el eje educación superior inclusiva con tres artículos que se aproximan a la temática, dos desde la exploración de nuevos itinerarios para la responsabilidad social universitaria, tanto desde el análisis de las políticas públicas encaminadas hacia las acciones afirmativas inclusivas, como desde la valoración de la experiencia en la implementación de las mismas, y otro artículo que aborda el análisis curricular y la formación universitaria en contextos de interculturalidad y migración.

El artículo Limites y posibilidades de las politicas institucionales inclusivas en las universidades del conurbano bonaerense. Explorando caminos nuevos desarrollado por Ana María Cambours de Donini, aborda las políticas institucionales que se implementan en las universidades públicas argentinas del área geográfica establecida, desde el ámbito de las políticas orientadas a contribuir al sostenimiento económico de los y las ingresantes y desde el ámbito del acompañamiento académico y la flexibilización de la propuesta curricular de oferta a distancia. El artículo realiza un breve recorrido por el escenario universitario argentino, resaltando la ampliación de las instituciones, las reformas y el debate entre masificación como herramienta para la reducción o para la reproducción de desigualdades sociales. Por otro lado, plantea el derecho a la educación superior, como bien público y derecho humano personal y social que se traduce en igualdad y gratuidad, mismas que en la práctica no aseguran la inclusión. El análisis de las políticas públicas se plantea desde cuatro líneas: bienestar estudiantil, experiencias de bimodalidad ante la heterogeneidad y la masificación, nuevos desafíos de pedagogía universitaria y editoriales universitarias y políticas de acceso a libros, como estrategias de acompañamiento a las políticas públicas inclusivas. Para finalizar, destaca la problemática vinculada a la sostenibilidad de acciones y programas afirmativos, a la falta de sinergia y continuidad a las iniciativas pedagógicas, a la falta de estudios sobre las editoriales universitarias y su rol en la democratización educativa, y a la necesidad de concreción de dichas iniciativas en políticas públicas que incidan en los procesos de inclusión educativa.

El artículo La educación inclusiva en instituciones formadoras de maestros. Balance de la experiencia de la Universidad Pedagógica Nacional de Colombia, desarrollado por Olga Cecilia Díaz, Luz Mary Lache, Isabel Garzón, Adolfo León Atehortúa, analiza las políticas institucionales, cambios normativos, estrategias y acciones implementadas por la Universidad Pedagógica Nacional de Colombia en relación a la educación inclusiva, a la formación de maestros y al rol del Estado como corresponsable de la implementación de las mismas. El artículo describe las políticas institucionales para favorecer la educación inclusiva, mismas que para su operacionalización se han incorporado a la planificación estratégica institucional, sin embargo, se señala el distanciamiento entre las aspiraciones enunciadas y la concreción y alcance de las metas. Dentro de los cambios normativos, estrategias y acciones descritas destacan las orientadas a la ampliación del alcance de la admisión inclusiva para abarcar tanto a los aspirantes procedentes de grupos étnicos, como a los 
aspirantes víctima del conflicto armado en Colombia; así como las orientadas a la reorganización de los procesos de selección y admisión. Los efectos de dichas medidas se traducen en el incremento de los estudiantes admitidos y matriculados y en variaciones en los índices de selectividad y absorción. Se concluye enunciando tres problemáticas vinculadas a la educación superior inclusiva: políticas inclusivas integrales y flexibles; educación superior inclusiva versus políticas de calidad; recursos, Estado y definición de fines e indicadores como medidores de la eficiencia educativa versus criterios para la equidad e inclusión.

El artículo Formación universitaria, migraciones e interculturalidad en España: Una revisión de la oferta educativa de los estudios de grado de educación infantil, educación primaria, pedagogía y educación social, desarrollado por María Rubio Gómez, Raquel Martinez Chicón y Antonia Olmos Alcaraz, presenta una revisión de la oferta educativa en el campo de las ciencias de la educación, de las universidades públicas de las comunidades autónomas españolas, a la luz de temas como migraciones e interculturalidad, multiculturalidad y diversidad cultural. Los resultados mostrados por los autores destacan que existe una oferta amplia de materias relacionadas con interculturalidad y migraciones, sin embargo, la mayoría son de naturaleza optativa y tienen bajo número de créditos académicos asignados, por lo que se refleja la poca visibilidad explícita de estas temáticas en la formación obligatoria, relegando a la optatividad lo que es una realidad a la que tendrán que enfrentarse los futuros formadores. Los autores concluyen que la capacidad de modificar el sistema educativo a partir de nuevas perspectivas y contenidos curriculares adaptados a la situación de multiculturalidad, actualmente es reducida, lo que incide en la falta de comprensión sobre la realidad multicultural existente. Por lo que proponen una educación con enfoque transversal intercultural que integre contenidos que promuevan una formación universitaria que fomente la convivencia multicultural, para una sociedad equitativa, justa, plural y diversa.

\section{La dimensión social de la educación superior: a modo de reflexión final}

Derivadas de la lectura de los diferentes artículos contenidos en el monográfico, pueden mencionarse algunas consideraciones importantes en relación con la dimensión social de la educación superior. Por un lado, este número monográfico presenta algunos atisbos a una aproximación a la conceptualización de la dimensión social de la educación superior, sin embargo, aún se requiere mayor profundización tanto sobre los elementos que la conforman, como sobre el contenido y abordaje de dichos elementos. Los ejes a través de los que transcurren las aportaciones: Características sociodemográficas y composición social; elección de estudios y matricula universitaria; dimensión social, universidad y sociedad; $y$, educación superior inclusiva nos ofrecen pistas relevantes sobre las temáticas que preocupan y ocupan a los/las investigadoras.

La dimensión social de la educación superior hace, tradicionalmente, referencia a los factores que determinan la composición de la matrícula universitaria (Villaroya Ariño, 2014) aunque como afirma Herrera (2018) se convierte en un elemento imprescindible para determinar las acciones destinadas a eliminar las barreras que impiden a la población acceder a la educación superior.

El abordaje desarrollado por las diversas contribuciones, alrededor de las características sociodemográficas y la composición social continúan manifestando la preocupación por la profundización en la desigualdad, la inequidad y los grupos tradicionalmente infrarrepresentados ya que la responsabilidad social, del rol de la universidad, del aprendizaje y de la cohesión social, devela la necesidad de incrementar y mejorar las prácticas y políticas que se están implementando en las instituciones de educación superior, para realmente alcanzar la aspiración de una educación verdaderamente inclusiva y democrática. Existen múltiples estudios que vinculan la ocupación de los/as ascendientes directos haciendo referencia al bac- 
kground familiar del cual proceden los estudiantes que acceden a la universidad y que terminan persistiendo. Los datos obtenidos en distintos estudios señalan la importancia de este factor, tanto por el nivel adquisitivo, como por el capital formativo y cultural (Baraño, Finkel y Rodríguez, 2011).

Las diversas contribuciones revelan la necesidad de desarrollar estudios e investigaciones que permitan dar mayores pasos hacia el conocimiento del perfil, las características y condiciones de los estudiantes como herramienta para el diseño de las políticas que les favorezcan; así como hacia el análisis del impacto de los procesos de masificación vividos en las universidades y hacia sus resultados, en términos de la reducción de las desigualdades y de la inclusión en el acceso, en la permanencia, en las condiciones de vida y en los resultados académicos de los estudiantes. Asimismo, se proponen estudios más íntimamente relacionados con la participación democrática, los servicios a estudiantes o sus motivaciones y modos de vida.

En concordancia con la agenda 2030 de Naciones Unidas, las preguntas relacionadas con la calidad de vida proporcionan información valiosa para reflexionar sobre los desafíos que plantea garantizar la plena igualdad de oportunidades en la educación superior. En los últimos 15 años la región latinoamericana ha presentado avances sustantivos en lo que respecta al acceso a las IES (Ferreyra, Avitabile, Botero Álvarez, Haimovich Paz, y Urzúa, 2017). No obstante, aún persisten enormes desigualdades relacionadas con las dinámicas de inequidad que impiden el éxito y la permanecía en el sistema educativo de los sectores más desfavorecidos de la población (Trucco, 2014). Desafíos que, en muchos casos, buscan mejorar además la permanencia de los/as estudiantes en la educación superior en tanto objetivo de justicia social que es la esencia de la pertinencia de la educación superior (Montané, A.; Nairdorf, J. Teodoro, A.; 2012) y que requiere conocer, apoyar e identificar las necesidades de los diferentes grupos de estudiantes.

\section{Referencias bibliográficas}

Barañano, Margarita; Finkel, Lucila y Rodríguez, Elena (2011): "Procedencia socio-familiar", capítulo 5 en Ariño, Antonio y Llopis, Ramón (dirs.). ¿Universidad sin clases? Condiciones de vida de los estudiantes universitarios en España (Eurostudent IV), pp. 87-116.Madrid: Ministerio de Educación.

Conferencia de Ministros Europeos responsables de Educación Superior. (2005). El Espacio Europeo de Educación Superior-Alcanzando las Metas. Bergen.

Conferencia de Ministros Europeos responsables de Educación Superior. (2007). Comunicado de Londres. Hacia el Espacio Europeo de Educación Superior: Respondiendo a los Retos de un Mundo Globalizado. Londres.

Conferencia de Ministros Europeos responsables de Educación Superior. (2009). El Proceso de Bolonia 2020: El Espacio Europeo de Educación Superior en la Nueva Década. Lovaina.

Naciones Unidas (2016). Agenda 2030 y los Objetivos de Desarrollo Sostenible Una oportunidad para América Latina y el Caribe. Santiago (en línea). Cepal. http://www.sela.org/media/2262361/agenda2030-y-los-objetivos-de-desarrollo-sostenible.pdf, consultado mayo 2019.

Trucco, Daniela. (2014). Educación y desigualdad en América Latina. Santiago de Chile: Cepal

Ferreyra, Marta; Avitabile, Ciro; Botero Álvarez, Javier.; Haimovich Paz, Francisco y Urzúa, Sergio (2017). Momento decisivo: La educación superior en América Latina y el Caribe. Washington DC: Grupo Banco Mundial. 
Damián Herrera Cuesta (2019): “¿Quién estudia en la universidad? La dimensión social de la universidad española en la segunda década del siglo xxı”. Revista de Sociología de la Educación (RASE), 12 (1).

Montané, Alejandra; Nairdorf, Judith y Teodoro, Antonio (2013). "Social and Cognitive Justice: The social Relevance of the Higher Education in Latin America" en Ira Bogotch. Shields International Handbook of Educational Leadership and Social (In)Justice. Springer International Handbooks of Education 29.

Villarroya Ariño, Antonio (2014): “La Dimensión Social en la Educación Superior”. Revista de la Asociación de Sociología de la Educación (RASE), 7 (1), 17-41. 